\title{
Formulation development and evaluation of colon targeted delayed release methotrexate pellets for the treatment of colonic carcinoma
}

\author{
Mansi Paradkar ${ }^{1 *}$, Jinal Amin² \\ ${ }^{1}$ Department of Pharmaceutics \& Pharmaceutical Technology, Ramanbhai Patel College of Pharmacy, Charotar University \\ of Science and Technology (CHARUSAT), Changa, Gujarat, India, ${ }^{2}$ Apothecon Pharmaceutical Pvt. Ltd., Padra-Jambusar \\ Highway, P.O-Dabhasa, Ta-Padra, Vadodara, Gujarat, India
}

\begin{abstract}
Colonic carcinoma is one of the most common internal malignancies and is the second leading cause of deaths in United States. Methotrexate (MTX) is a drug of choice in the treatment of colon cancer. The aim of the present research work was to develop and characterize colon targeted pellets of MTX for treatment of colonic carcinoma. The product and process parameters were optimized by screening methods. Pellets were prepared by extrusion spheronization using microcrystalline cellulose (MCC) as spheronizing aid and ethyl cellulose (EC) as release retardant in different ratio. Based on the physical appearance, sphericity and \% in vitro drug release, batch P17 containing EC: MCC (3:7) was optimized for core pellets. The site specificity was obtained by screening the coating polymers and by coating the core pellets with EudragitS100. The $3^{2}$ full factorial design was applied in which airflow rate (X1) and coating time (X2) were the independent parameters and physical appearance (Y1) and time taken for $100 \%$ drug release (Y2) were selected as the dependent variables. From the results obtained, $6 \mathrm{~min}$ of coating time and $60 \mathrm{~cm}^{3} / \mathrm{min}$ airflow rate was optimized. The batch B5 showed appropriate physical appearance and $\%$ in vitro drug release upto $17 \mathrm{hr}$ indicating sustained release property. The ex-vivo studies performed on rat colon indicated a significant relation with the in vitro drug release. The drug release followed Higuchi's model indicating the diffusion pattern of drug release from the matrix of pellets. Thus, the coated pellets can be a good candidate for site specific delivery of MTX to colon by decreasing the gastric irritation and thus to improve bioavailability.
\end{abstract}

Keywords: Ethyl cellulose. Microcrystalline cellulose. Eudragit S100. Extrusion-spheronization. Modified fluid bed coater. Ex-vivo drug release study.

\section{INTRODUCTION}

Colon cancer is a cancer from uncontrolled cell growth in the colon. Colon cancer cells invade and damage healthy tissue in the nearby region. Chemotherapy is used for the treatment of colonic carcinoma. Conventional chemotherapy is not effective in colonic carcinoma as the drug normally dissolves and absorbs in the stomach and small intestine and does not reach the target site in effective concentration. Thus, effective treatment demands site specific targeting of the drug for more effective treatment.

\footnotetext{
*Correspondence: M. Paradkar. Department of Pharmaceutics \& Pharmaceutical Technology, Ramanbhai Patel College of Pharmacy, CHARUSAT, Changa, Anand-388 421, Gujarat, India. Contact number: +917874379184. E-mail: mansiparadkar.ph@charusat.ac.in
}

Methotrexate (MTX) is an antineoplastic, folate antimetabolite with immunosuppressant properties which is used as the drug of choice in the treatment of colonic carcinoma. It is an inhibitor of tetrahydrofolate dehydrogenase and prevents the formation of tetrahydrofolate, necessary for synthesis of thymidylate, an essential component of DNA. Methotrexate is used in the treatment of certain neoplastic diseases, severe psoriasis, and adult rheumatoid arthritis and in autoimmune diseases such as Crohn's disease, etc. Lynch syndrome, often called hereditary nonpolyposis colorectal cancer, is a type of inherited cancer of the digestive tract, particularly the colon and the rectum. The $40 \%$ of lynch syndrome related colorectal cancers are caused by inherited mutations in the MSH2 gene. Methotrexate selectively destroys the cells lacking the MSH2 gene function and it is the excellent 
treatment for patients with genetic alteration (Sogali, Yousuff, Nayak, 2012).

It is possible to deliver MTX to the inner surface of the colon that destroys small tumours which arise spontaneously in this region by the development of $\mathrm{pH}$ dependent system. The even drug distribution through colon is possible by multiparticulate system. The enteric coated pellets cover the entire region of colon and resist the release of the drug in stomach and small intestine and thereby preventing the irritation in the stomach. Thus, the aim of the present work was to formulate and evaluate delayed release colon targeted pellets of MTX for the treatment of colonic carcinoma by decreasing the gastric irritation, reducing dose frequency and to improve patient compliance.

\section{MATERIAL AND METHODS}

Methotrexate (Sun Pharmaceutical Pvt. Ltd, Baroda, Gujarat), Eudragit S100 and Eudragit RS30D (Rohm Pharma, GmbH, Germany) and Chitosan (Chitoclear FG95) (Primex, Norway) were obtained as gift samples. Microcrystaline cellulose (Avicel PH101), pectin and carragenan were procured from FMC Biopolymer, USA and Himedia, India respectively. Ethyl cellulose was obtained from Loba Chemie, India. All ingredients and reagents used were of analytical or equivalent grade.

\section{Formulation development of Methotrexate core pellets}

\section{Pre-formulation Studies}

\section{Selection of excipients}

The excipients were selected based on the literature review (Sogali, Yousuff, Nayak, 2012; Vervaet, Baert, Remon, 1995; Steckel, Mindermann-Nogly, 2004; Priese, Frisch, Wolf, 2015). Pectin, carrargenen, chitosan, ethyl cellulose as release retardants and microcrystalline cellulose (MCC) (Avicel PH 101) as the spheronizing aid were selected for the preformulation studies.

Method of preparation of core pellets (Gupta et al., 2011)

The pellets were prepared by pelletization technique using extrusion/spheronization. All excipients were passed through sieve No. 40 prior to pelletization and then they were mixed uniformLy in a mortar and pestle. Granulating fluid was added dropwise to the mixture to obtain damp mass which was then extruded using a screw extruder. The extrudates were immediately spheronized and the pellets were dried in fluid bed dryer.

Screening of polymers (Sogali, Yousuff, Nayak, 2012; Steckel, Mindermann-Nogly, 2004)

The formation of pellets using different polymers and their concentration was taken into account. The batches were prepared by taking ratio of 1:9 to $9: 1$ of polymer: MCC. The polymer was selected depending on the physical appearance considering whether pellets were formed or not and its sphericity. The batches were prepared without incorporation of drug. The polymer which formed pellets with appropriate sphericity and physical appearance was selected for further study.

\section{Determination of the granulating fluid and its amount}

The isopropyl alcohol (IPA) and water were selected as the granulating fluids on the basis of literature review (Hileman, Upadrashta, Neau, 1997). The granulating fluid was added in batches P1 (IPA) and P2 (water) till it formed the proper pellets by keeping all the other product parameters constant. The amount of the selected granulating fluid was varied and depending upon the formation of pellets and the physical appearance, the optimum amount of granulating fluid was determined.

\section{Optimization of process variables}

Various process variables like spheronization speed, spheronization time and drying time were optimized by formulation of different batches as per Table I. The fixed ratio of Ethyl cellulose: MCC were used for preparation of pellets for the optimization. Drug was not incorporated in these batches and the other parameters were kept constant.

\section{Formulation of Methotrexate core pellets}

Based on the results of the preformulation studies including selection and the optimization of the product and process parameters, the different batches P15-P23 containing ethyl cellulose: MCC (1:9 to 9:1) were formulated with the incorporation of Methotrexate as a drug. The selection of the optimized batch of core pellets was performed based on the results of formation of pellets, physical appearance, sphericity, micromeritic parameters, $\%$ drug loading and the $\%$ drug release. 
TABLE I - Optimization of various process variables for pellet formulation

\begin{tabular}{|c|c|c|c|c|c|c|c|c|c|}
\hline Batch no. & P6 & P7 & P8 & P9 & P10 & P11 & P12 & P13 & P14 \\
\hline Spheronization speed & $1000 \mathrm{rpm}$ & $1150 \mathrm{rpm}$ & $1350 \mathrm{rpm}$ & $1150 \mathrm{rpm}$ & $1150 \mathrm{rpm}$ & $1150 \mathrm{rpm}$ & $1150 \mathrm{rpm}$ & $1150 \mathrm{rpm}$ & $1150 \mathrm{rpm}$ \\
\hline Spheronization time & $\begin{array}{l}\text { Till pellets } \\
\text { were } \\
\text { formed }\end{array}$ & $\begin{array}{l}\text { Till pellets } \\
\text { were } \\
\text { formed }\end{array}$ & $\begin{array}{l}\text { Till pellets } \\
\text { were } \\
\text { formed }\end{array}$ & $3 \mathrm{~min}$ & $6 \mathrm{~min}$ & $9 \mathrm{~min}$ & $6 \mathrm{~min}$ & $6 \mathrm{~min}$ & $6 \min$ \\
\hline Drying time & $\begin{array}{l}\text { Till pellets } \\
\text { were dried }\end{array}$ & $\begin{array}{l}\text { Till pellets } \\
\text { were dried }\end{array}$ & $\begin{array}{l}\text { Till pellets } \\
\text { were dried }\end{array}$ & $\begin{array}{l}\text { Till pellets } \\
\text { were dried }\end{array}$ & $\begin{array}{l}\text { Till pellets } \\
\text { were dried }\end{array}$ & $\begin{array}{l}\text { Till pellets } \\
\text { were dried }\end{array}$ & $2 \mathrm{~min}$ & $3 \mathrm{~min}$ & $4 \mathrm{~min}$ \\
\hline
\end{tabular}

Evaluation of Methotrexate core pellets (Steckel, Mindermann-Nogly, 2004; Gupta et al., 2011; Akhgari, Sadeghi, Garekani, 2006; Kaynak, Kas, Oner, 2007; He et al., 2008; Sriamornsak et al., 2008)

\section{Micromeretic parameters}

The micromeretic parameters of the uncoated pellets comprised the angle of repose, bulk density, tapped density, Compressibility Index and Hausner's ratio.

\section{Angle of repose $(\theta)$}

It is used to estimate the flow property of pellets. The angle of repose of pellets was determined by the funnel method. The accurately weighed pellets were taken in the funnel. The height of the funnel was adjusted in such a way that the tip of the funnel remains $2 \mathrm{~cm}$ above the base. The pellets were allowed to flow through the funnel freely on to the surface. The diameter of the pellet cone was measured and angle of repose was calculated using the following equation.

$$
\tan \theta=\frac{h}{r}
$$

where $\mathrm{h}=$ Height $(\mathrm{cm}), \mathrm{r}=$ Radius $(\mathrm{cm})$ of the pellet cone .

\section{Bulk density}

Apparent bulk density was determined by pouring the pellets into a graduated cylinder. The bulk volume and weight of the pellets was determined. The bulk density was calculated using following equation,

$$
\rho b=\frac{M}{V_{b}}
$$

where, $\rho b=$ Bulk density $\left(\mathrm{gm} / \mathrm{cm}^{3}\right), \mathrm{M}=$ Weight of pellets $(\mathrm{gm}), \mathrm{V}_{\mathrm{b}}=$ Bulk volume $(\mathrm{mL})$

\section{Tapped density}

The measuring cylinder containing a known mass of pellets was tapped for 100 times. The minimum volume occupied in the cylinder and weight of the pellets was measured. The tapped density was calculated using following equation,

$$
\rho t=\frac{M}{V_{t}}
$$

where, $\rho t=$ Tapped density (gm. $/ \mathrm{cm} 3), \mathrm{M}=$ Weight of pellets (gm.), $\mathrm{V}_{\mathrm{t}}=$ Tapped volume $(\mathrm{mL})$

\section{Hausner's ratio}

The Hausner's ratio is used for the estimation of the flow property of pellets. Hausner's ratio is the ratio of tapped density to bulk density of pellets. Its value less than 1.25 indicates excellent flow of particles and value more than 1.25 indicates poor flow property. The Hausner's ratio of the granules was determined by the equation,

$$
\text { Hausner's ratio } H p=\frac{\rho t}{\rho b}
$$

\section{Moisture content}

During extrusion, three samples of about 8-10 gm were taken and dried at $75^{\circ} \mathrm{C}$ over $36 \mathrm{hr}$ in a hot air oven. The moisture content (Mc) (\%) was calculated according to the equation, where $m_{d}$ is the dried mass and $m_{w}$ is the wet mass:

$$
\mathrm{Mc}=\left(\mathrm{m}_{\mathrm{w}}-\mathrm{m}_{\mathrm{d}}\right) / \mathrm{m}_{\mathrm{d}} * 100
$$

\section{Sphericity index}

In order to determine the sphericity of the pellets, the pellets were taken on a slide. The slide was placed on the stage of the microscope and the images and the circulatory parameters were determined. This parameter was checked 
by taking 100 pellet particles. The sphericity index was calculated using the equation;

$$
\mathrm{S}=\mathrm{p} 2 /(12.56 \times \mathrm{X})
$$

where, $\mathrm{A}$ is the area $\left(\mathrm{cm}^{2}\right)$ and $\mathrm{p}$ is the perimeter $(\mathrm{cm})$

\section{Drug content}

Equivalent weight to $2.5 \mathrm{mg}$ of methotrexate pellets was dissolved in $50 \mathrm{~mL}$ of $0.1 \mathrm{~N} \mathrm{HCl}$. The drug concentration in $0.1 \mathrm{~N} \mathrm{HCl}$ with proper dilution was analyzed spectrophotometrically by UV spectrophotometer at $306 \mathrm{~nm}$.

\section{Friability}

Friability of all pellets was determined by USP friability test. Friability of the pellet formulations was evaluated over $10 \mathrm{~g}$ of samples in Roche Friabilator at 25 rpm for $4 \mathrm{~min}$. Prior to and following the test, the weights of the formulations were accurately recorded and the friability ratios were calculated with Equation no. 7. The results were expressed in terms of the percentage of weight lost during the process.

$$
\mathrm{F}=\left(\mathrm{W}_{1}-\mathrm{W}_{2}\right) / \mathrm{W}_{1} * 100
$$

where, $\mathrm{W}_{1}$ is the initial weight, $\mathrm{W}_{2}$ is the final weight of the formulation.

\section{In vitro drug release studies of uncoated pellets (Gupta et al., 2011)}

The release of drug from the developed formulations in the environment of colon was determined using USP XXIII dissolution apparatus I. Pellets were placed in the basket, which was further immersed in beaker containing $900 \mathrm{~mL}$ of phosphate buffer $\mathrm{pH} 7.4$ as dissolution media maintained at $37 \pm 0.5^{\circ} \mathrm{C}$ and $50 \mathrm{rpm}$. Aliquots of 5 $\mathrm{mL}$ were withdrawn every $15 \mathrm{~min}$ for first $\mathrm{hr}, 30 \mathrm{~min}$ for second hr and then every hour until $100 \%$ drug release was obtained, with the replacement of $5 \mathrm{~mL}$ of the fresh medium. Correction factors for each aliquot were considered in calculation of drug release profile. Absorbance of sample after proper dilution was measured at $303 \mathrm{~nm}$ using U.V. spectrophotometer against blank. Concentration of drug was determined from the standard plots of the drug in phosphate buffer $\mathrm{pH} 7.4$ previously calculated and the $\%$ drug release was calculated at each sampling time. The study was performed in triplicates.

\section{Formulation of enteric coated Methotrexate pellets}

The optimized batch of core pellets was selected for the coating. The pellet cores were coated in a fabricated fluidized bed coater (Figure 1). The air flow rate and the coating time were controlled and the pellets were dried in the fluid bed dryer which were then evaluated for measurement of the physical characteristics.

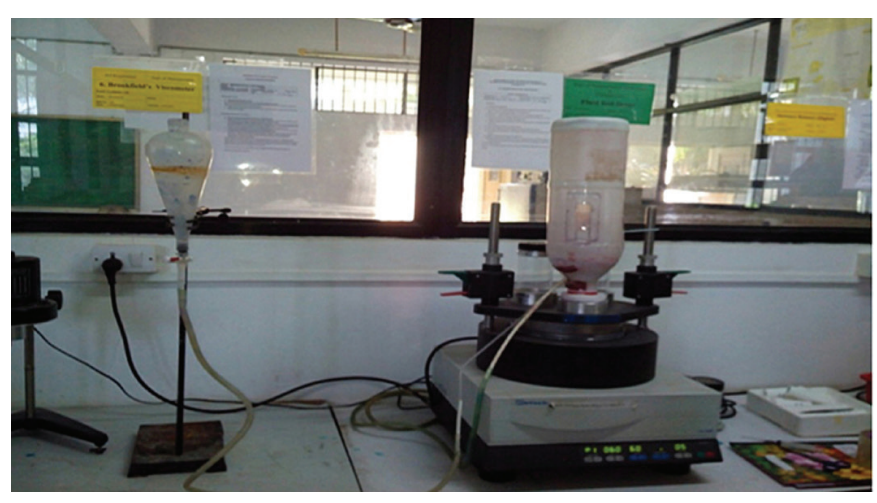

FIGURE 1 - Fabricated fluid bed coater.

\section{Screening of coating polymer and its amount} (Akhgari, Sadeghi, Garekani, 2006; Kaynak, Kas, Oner, 2007; He et al., 2008)

Based on the literature review, coating polymers were selected. Eudragit FS30D (ready to use dispersion) and Eudragit S100 were dissolved in organic solvent (IPA: Acetone) and then PEG 6000 as plasticizer and talc were added to prepare the coating solution. The pellets of batch C1 (Eudragit FS30D) and C2 (Eudragit S100) were coated to select the appropriate coating polymer formed by fabricated fluid bed coater to obtain good coating uniformity. The amount of coating polymer was varied in batch C3, C4 and C5 (Table II) and the selection was done based on the physical appearance and the coating consistency of the pellets.

\section{Determination of coating bed temperature}

The temperature at which the pellets are dried during and after coating process is the important parameter for the coated pellets. The pellets of batches C6-C8 were dried at different temperatures (Table II). The selection was performed on the basis of whether the pellets got dried at that particular temperature or not and the physical appearance that includes the coating consistency and drying of the film coat. 
TABLE II - Optimization of coating variables of methotrexate core pellets

\begin{tabular}{lcccccc}
\hline Batch no. & C3 & C4 & C5 & C6 & C7 & C8 \\
\hline Amount of coating solution $(\mathrm{mL})$ & 5 & 7 & 9 & 7 & 7 & 7 \\
Coating bed temperature $\left({ }^{\circ} \mathrm{C}\right)$ & Till it dried & Till it dried & Till it dried & 50 & 60 & 70 \\
\hline
\end{tabular}

\section{Optimization of enteric coated methotrexate pellets}

The $3^{2}$ full factorial design was applied using Design Expert ${ }^{\circledR}$ 9.0.1.0 (Stat-Ease. Inc. Minneapolis, USA) software for the optimization of coated pellets. The air flow rate (X1) and the coating time (X2) were selected as independent variables and Physical characteristics such as properly coated pellets with no aggregates (Y1) and time required for $100 \%$ Drug release (Y2) were selected as the dependent variables (Table III). Results obtained were statistically analysed at 5\% level of significance. A check point analysis was performed to confirm the role of derived polynomial equation and contour plots in predicting the responses in the coating of the pellet formulation. Coating was performed experimentally at 2 points and then evaluated for the responses. Differences of theoretically computed values of dependent variables and experimentally obtained values of dependent variables were checked by using $t$ test. The desirability function was also applied to the responses.

\section{Evaluation of methotrexate coated pellets}

The different batches of Methotrexate coated pellets (Batches B1-B9) were evaluated by all the parameters as that of the uncoated pellets.

\section{In-vitro drug release studies of coated pellets} (Gandhi, Kaul, Panchagnula, 1999; Gupta et al., 2011)

The release of drug from the developed formulations in the environment of gastrointestinal tract was determined by USP XXIII dissolution apparatus I. Pellets were placed in the basket, which was further immersed in beaker containing $900 \mathrm{~mL}$ of dissolution media maintained at 37 $\pm 0.5^{\circ} \mathrm{C}$ and $50 \mathrm{rpm}$. For cumulative drug release studies, dissolution media consisted of $0.1 \mathrm{~N} \mathrm{HCl}$ was kept for $2 \mathrm{~h}$, which was further placed in phosphate buffer $\mathrm{pH}$ 6.8 for $4 \mathrm{~h}$ and then in phosphate buffer $\mathrm{pH} 7.4$ for $16 \mathrm{~h}$. Aliquot samples of $5 \mathrm{~mL}$ were withdrawn every $15 \mathrm{~min}$ for first hour, $30 \mathrm{~min}$ for second hour and then every hour up to $16 \mathrm{~h}$ with replacement of $5 \mathrm{~mL}$ of the fresh medium. Correction factors for each aliquot were considered in the calculation of release profile. Absorbance of sample after proper dilution was measured at $303 \mathrm{~nm}$ using U.V. spectrophotometer against blank. Concentration of drug was determined from the standard plots of the drug in phosphate buffer $\mathrm{pH} 7.4$ previously calculated and the \%drug release was calculated at each sampling time. The study was performed in triplicates. To study the release mechanism of the pellets formulation, the release data were fitted to the different kinetics models.

Ex-vivo study (He et al., 2008)

Male Albino Wister rats were used for the pharmacokinetic study (Protocol No. RPCP/IAEC/20132014/MPH-PT-42). The rat was kept in the fasted condition over night. It was then sacrificed and the colon along with its contents was isolated. The lower end was closed by using thread or clip while from the upper end pellets equivalent to unit dose were placed and the upper end was also tied. The whole tissue was then placed in the assembly containing phosphate buffer $\mathrm{pH} 7.4$ and continuous air supply was provided with the maintenance of temperature. Samples were withdrawn at every hour and analysis was performed by UV spectrophotometer at $303 \mathrm{~nm}$.

TABLE III - $3^{2}$ full factorial design for methotrexate coated pellets

\begin{tabular}{|c|c|c|c|c|}
\hline \multirow{2}{*}{$\begin{array}{c}\text { Factors } \\
\text { (Independent variables) }\end{array}$} & \multicolumn{3}{|c|}{ Levels Used } & \multirow{2}{*}{$\begin{array}{c}\text { Responses } \\
\text { (Dependent variables) }\end{array}$} \\
\hline & -1 & $\mathbf{0}$ & 1 & \\
\hline $\mathrm{X} 1=$ Air Flow rate $\left(\mathrm{cm}^{3} / \mathrm{min}\right)$ & 40 & 60 & 80 & Y1=Physical characteristics* \\
\hline $\mathrm{X} 2=$ Coating time $(\mathrm{min})$ & 4 & 6 & 8 & $\mathrm{Y} 2=\%$ Drug release \\
\hline
\end{tabular}

*Physical appearance: 1- Aggregated pellets, 2- Average coating with some aggregated pellets, 3- Properly coated pellets with no aggregates 


\section{RESULTS AND DISCUSSION}

\section{Formulation development of methotrexate core pellets}

\section{Pre-formulation studies}

Screening of polymers

During screening of polymers, the pellets were not formed in any ratio from 1:9 to 9:1 (Pectin: MCC). This might be due to the fact that pectin imbibed a lot of water and did not break up to form pellets. In the batches containing carrageenan, the pellets were formed in ratio 1:9 to 4:6 (carrageenan: MCC) but proper spheres were not formed; they formed rough surface and uneven shaped pellets whereas in other ratios pellets were not formed. In the batches containing chitosan, proper even shaped pellets were formed with consistent sphericity in ratios 1:9 to 5:5 (chitosan: MCC) but pellets got immediately easily disintegrated because chitosan acted as pore forming agent (Omwancha et al., 2013). Therefore, pellets with higher concentration of chitosan were not prepared. This might be due to weak ionic bond between chitosan and MTX (Gupta et al., 2011; Steckel, Mindermann-Nogly, 2004) and thus it was not continued for the further study. In the batches containing ethyl cellulose, pellets were properly formed in ratio $1: 9$ to 5:5. Based on the results, it was concluded that ethyl cellulose forms appropriate pellets and so it was selected as appropriate release retardant for the pellet preparation (He et al., 2008). Out of all these batches, randomLy ratio 2:8 (ethyl cellulose: MCC) was selected for the optimization of the other parameters.

\section{Determination of the granulating fluid and its amount}

From the results, it was concluded that when isopropyl alcohol was used as granulating fluid, no binding took place. The addition of high amount of the fluid lead to formation of a partially damp mass which again turned into powder upon extrusion due to its volatile nature.

In case of water, appropriate binding occurred as the water molecules properly get adsorbed onto the surface and so upon extrusion, appropriate extrudates were formed. In addition, non-volatile nature of water helps in retaining the sphericity upon spheronization(Vervaet, Baert, Remon, 1995). Therefore, water was selected as the appropriate granulating liquid. From various trials, $12 \mathrm{~mL}$, $14 \mathrm{~mL}$ and $16 \mathrm{~mL}$ of the granulating fluid were selected as the optimum range. In batch $\mathrm{P} 3(12 \mathrm{~mL})$, the damp mass formed was comparatively dry and upon extrusion only very few amount of the extrudates were formed and upon spheronization they remained as extrudates, due to lack of moisture. In batch P4 (14 mL) of water, proper damp mass was formed and also the extrudates were properly formed. When these extrudates were spheronized, they lead to the formation of proper pellets with appropriate sphericity. In batch P5 (16 mL) of water, highly damp mass was formed and extrudates tend to aggregate and stick to each other upon extrusion and when these extrudates were spheronized, it lead to the formation of dumbled shaped pellets. Thus, batch $\mathrm{P} 4$ containing $14 \mathrm{~mL}$ of granulating fluid was considered as optimum for the further study.

Optimization of the process parameters (Vervaet, Baert, Remon, 1995; Gandhi, Kaul, Panchagnula, 1999)

The optimization of the process variables was performed by selecting the $\mathrm{P} 4$ batch. The process parameters like extrusion speed, extrusion time, spheronization time, spheronization speed and drying time were selected. The extrusion speed and extrusion time did not have considerable effect on the pellet properties so they were fixed.

\section{Spheronization speed}

The spheronization speed is a critical parameter for pellet formulation (Vervaet, Baert, Remon, 1995). In the batch $\mathrm{P} 6$, the extrudates were spheronized at $1000 \mathrm{rpm}$ till it formed pellets. The pellets formed were more of rod and dumbled shaped and upon increasing time, the pellet got dried and so they did not turn into appropriate spheres. This can be due to the fact that the extrudates got partially dried and so they remained rods even after high time of spheronization. Batch P7 was spheronized at 1150 $\mathrm{rpm}$ till the pellets were formed. The extrudates properly got converted to pellets and perfect spheres were formed. In batch P8, the extrudates were spheronized at 1350 rpm till they formed pellets but there was wide range of particle size variation and an increased amount of fines. Therefore, batch P7 prepared with $1150 \mathrm{rpm}$ was selected as the optimum batch.

\section{Spheronization time}

Spheronization time is also an important parameter as lower and higher spheronization time leads to aggregation of particles which do not retain their sphericity upon drying (Vervaet, Baert, Remon, 1995). In batch P9 (3 min), pellets were formed but the sphericity was not obtained. The pellets formed were partially dumbled and 
rod shaped. The batch P10 (6 min) exhibited pellets with proper sphericity while in batch P11 (9 min), the pellets were formed but they tend to re-aggregate. Also upon drying, there was a large variation in size distribution and increased amount of fines. Thus, $6 \mathrm{~min}$ spheronization time was selected for the preparation of spherical pellets with least amount of fines during further study.

\section{Drying time}

Proper drying is required for the perfect pellet formation as moisture content may lead to fungal growth and excess of drying may lead to blackening of pellets (Akhgari, Sadeghi, Garekani, 2006).

In the batch P12 with 2 min of drying time, the pellets did not dry properly as they tend to aggregate due to high moisture content. In batch P13, the drying of the pellets was appropriate while in the batch $\mathrm{P} 14$ having drying time of $9 \mathrm{~min}$, over drying took place. So batch P13 with drying time of 6 min was selected for further study.

Evaluation parameters of the methotrexate core pellets (Akhgari, Sadeghi, Garekani, 2006; Steckel, Mindermann-Nogly, 2004; Kaynak, Kas, Oner, 2007; Gandhi, Kaul, Panchagnhla, 1999; Gupta et al., 2011)

During the formulation of batches P15-P23, in the batches P20-P23, the pellets were not formed. It led to formation of the white spots on the pellet surface with more of dumble shaped pellets. This can be attributed to the uneven distribution of ethyl cellulose and a considerable decrease in the spheronizing aid, MCC (Akhgari, Sadeghi, Garekani, 2006) (Table IV). From table V, it was concluded that batch P17 had good flow property as compared to other batches. The batches P15 to P19 exhibited sphericity index ranging from 0.9967 to 0.9980 . The ideal sphericity index of pellets should be near to 1 . Thus the sphericity index value near to 1 indicated that the prepared pellets exhibited proper sphericity and retained their structure with narrow size distribution (Figure 2). The moisture content helps to determine the presence of water in the extrudates as well as the pellets. Friability determines the strength of the pellets while the drug loading determines the amount of the drug present in the pellets (Kaynak, Kas, Oner, 2007; Jia et al., 2011). From the results, it was concluded that there was no specific marked difference in the values of each parameter. All the physical parameters were satisfactory of batches P15-P19 of ethyl cellulose and so the drug release study was carried out in those batches only. In batches P15-P19, the batch P17 exhibited 100\% drug release at $14^{\text {th }}$ hour which was found to be highest as compared to other batches. So batch P17 was selected the optimized batch (Figure 3). Thus, it can be concluded that as the polymer concentration increases, there is an increase in the sustain action of drug release. Though it

TABLE IV - Evaluation of the methotrexate core pellets

\begin{tabular}{lccccccccc}
\hline Batch no. & P15 & P16 & P17 & P18 & P19 & P20 & P21 & P22 & P23 \\
\hline Ethyl cellulose : MCC & $1: 9$ & $2: 8$ & $3: 7$ & $4: 6$ & $5: 5$ & $6: 4$ & $7: 3$ & $8: 2$ & $9: 1$ \\
Pellets formed & Yes & Yes & Yes & Yes & Yes & No & No & No & No \\
Sphericity obtained & Yes & Yes & Yes & Yes & Yes & No & No & No & No \\
100\% drug release & $11 \mathrm{~h}$ & $12 \mathrm{~h}$ & $14 \mathrm{~h}$ & $12 \mathrm{~h}$ & $11 \mathrm{~h}$ & - & - & - & - \\
\hline
\end{tabular}

TABLE V - Evaluation parameters of the methotrexate core pellets

\begin{tabular}{lccccc}
\hline Evaluation Parameters & P15 & P16 & P17 & P18 & P19 \\
\hline Bulk Density $(\mathrm{g} / \mathrm{mL})$ & $0.628 \pm 0.09$ & $0.634 \pm 0.07$ & $0.627 \pm 0.01$ & $0.659 \pm 0.03$ & $0.631 \pm 0.06$ \\
Tapped density $(\mathrm{g} / \mathrm{mL})$ & $0.729 \pm 0.05$ & $0.741 \pm 0.06$ & $0.727 \pm 0.02$ & $0.762 \pm 0.03$ & $0.734 \pm 0.05$ \\
Hausner's Ratio & $1.16 \pm 0.03$ & $1.16 \pm 0.02$ & $1.15 \pm 0.01$ & $1.15 \pm 0.03$ & $1.16 \pm 0.05$ \\
Angle Of Repose $(\theta)$ & $31.21 \pm 0.06$ & $30.14 \pm 0.04$ & $28.83 \pm 0.02$ & $31.27 \pm 0.03$ & $31.15 \pm 0.03$ \\
Moisture content of extrudates $(\%)$ & $4.98 \pm 0.09$ & $3.54 \pm 0.07$ & $3.63 \pm 0.03$ & $3.87 \pm 0.03$ & $3.98 \pm 0.08$ \\
\% Moisture content of pellets & $0.043 \pm 0.02$ & $0.049 \pm 0.01$ & $0.041 \pm 0.01$ & $0.047 \pm 0.02$ & $0.047 \pm 0.04$ \\
\% Friability & $0.421 \pm 0.02$ & $0.418 \pm 0.03$ & $0.415 \pm 0.01$ & $0.435 \pm 0.04$ & $0.513 \pm 0.03$ \\
\% drug loading & $99.53 \pm 0.02$ & $99.59 \pm 0.03$ & $99.62 \pm 0.01$ & $99.52 \pm 0.04$ & $99.60 \pm 0.03$ \\
\hline
\end{tabular}


was noticed that above the optimum concentration, the sustained action was decreased (He et al., 2008).
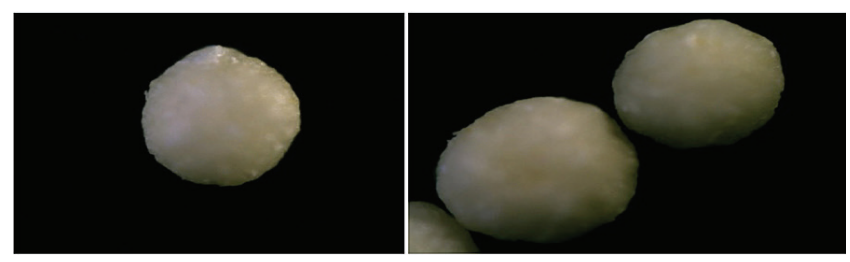

FIGURE 2 - Images of optimized batch (P17) of methotrexate core pellets.

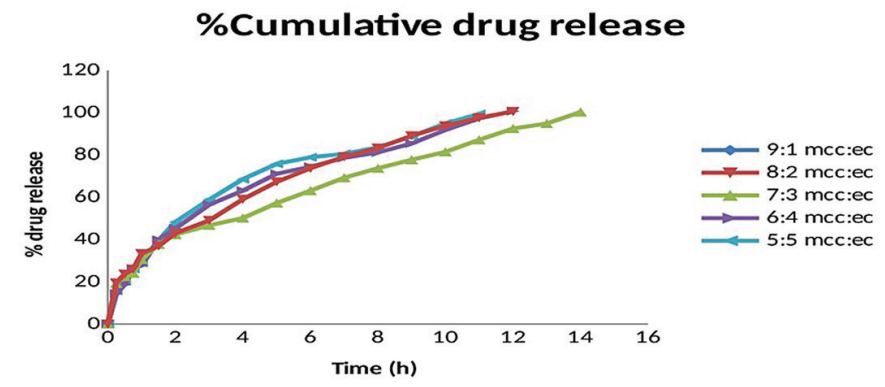

FIGURE 3 - \% Cumulative drug release of methotrexate core pellets.

Thus, the batch P17 with ethyl cellulose: MCC (3:7) ratio was selected as the optimized batch of core pellets and it was used further for coating of the pellets.

\section{Formulation of enteric coated methotrexate pellets}

\section{Optimization of coating parameters}

\section{Screening of coating polymer}

From the literature review and from the study of colonic pH, Eudragit FS30D (30\% dispersion) and Eudragit S100 were selected as the coating polymer for targeting the delivery of pellets to colonic site. In the batch C1 containing Eudragit FS30D, the improper coating was observed. The formed pellets got aggregated and proper coating film was not developed over pellets.

While in batch C2 containing Eudragit S100, even coating with flexible smooth surface was observed upon drying. Thus from the results, it was concluded that Eudragit S100 solution was optimum as it exhibited appropriate strength and flexibility to the pellet coat. This might be due to the fact that Eudragit S100 enables even coat and dries easily due to the presence of the organic solvents like acetone and iso-propyl alcohol (Akhgari et al., 2006) while Eudragit FS30D being an aqueous dispersion does not get dried easily and even small amount of it leads to aggregation of the pellet particles.

\section{Determination of amount of coating polymer}

Appropriate amount of coating solution is required for proper coating of pellets. In the batch $\mathrm{C} 3(5 \mathrm{~mL})$, the coating was uniform visually but under the microscope, large patches of uneven coat were observed. In the batch C4 (7 mL), coating was proper and the release of drug occurred only after coming in contact with colonic fluid. The batch C5 ( $9 \mathrm{~mL})$ exhibited proper coating but the drug release was sustained at a greater extent such that the drug was not released up to $2 \mathrm{~h}$ even at colonic $\mathrm{pH}$. Thus, the batch C4 was selected for further study (Table VI).

\section{Determination of coating bed temperature}

The coating bed temperature is the temperature at which the pellets get heated in the fabricated fluidized bed coater. In the batch $\mathrm{C} 6$, the pellets coated at $50{ }^{\circ} \mathrm{C}$ remained sticky which indicates too less temperature to dry the pellet while batch $\mathrm{C} 7$ prepared at $60^{\circ} \mathrm{C}$ exhibited proper drying of the coating film. In the batch $\mathrm{C} 8$, the polymer got melted during coating and hard aggregates were formed on cooling which might be due to higher temperature of the bed of coater $\left(70^{\circ} \mathrm{C}\right)$. Thus, batch $\mathrm{C} 7$ having $60{ }^{\circ} \mathrm{C}$ was considered as appropriate temperature for the coating process (Table VI).

\section{Statistical analysis by $3^{2}$ full factorial design}

$3^{2}$ full factorial design was applied for the optimization of methotrexate coated pellets. The results of the effect of the independent variables on dependent variables are given in following full model polynomial equation (equation 8 and 9).

Effect on time required for $100 \%$ drug release:

TABLE VI - Optimization of the coating variables

\begin{tabular}{lcccccc}
\hline Batch no. & C3 & C4 & C5 & C6 & C7 & C8 \\
\hline Amount of coating solution $(\mathrm{mL})$ & 5 & 7 & 9 & 7 & 7 & 7 \\
Coating bed temperature $\left({ }^{\circ} \mathrm{C}\right)$ & Till it dried & Till it dried & Till it dried & 50 & 60 & 70 \\
\hline
\end{tabular}


$\mathrm{Y}_{1}=16.78+0.33 * \mathrm{X}_{1}(\mathrm{p}=0.04634)-0.17 * \mathrm{X}_{2}(\mathrm{p}=0.7316)$

$+0.25 * \mathrm{X}_{1} \mathrm{X}_{2}(\mathrm{p}=0.03741)+0.67 * \mathrm{X}_{1}^{2}(\mathrm{p}=0.0468)+$

$2.17 * \mathrm{X}_{2}^{2}(\mathrm{p}=0.00598)$

Effect on physical appearance:

$\mathrm{Y}_{2}=2.67-4.93 * \mathrm{X}_{1}(\mathrm{p}=0.0583)+0.78 * \mathrm{X}_{2}(\mathrm{p}=0.6608)$

$+6.25 * \mathrm{X}_{1} \mathrm{X}_{2}(\mathrm{p}=0.04956)-0.167 * \mathrm{X}_{1}^{2}(\mathrm{p}=0.0234)-$

$6.12 * \mathrm{X}_{2}^{2}(\mathrm{p}=0.3982)$

The term of full model polynomial equation having insignificant $p$ value $(p>0.05)$ have negligible contribution to obtain dependent variables and thus are omitted to get reduced model equation.

The equation 10 and 11 representing the quantitative effect of the formulation variable on the Particle size and $\%$ cumulative drug release are describe below:

$\mathrm{Y}_{1}=16.78+0.33 * \mathrm{X}_{1}+0.25 * \mathrm{X}_{1} \mathrm{X}_{2}+0.67 * \mathrm{X}_{1}^{2}+$ $2.17 * \mathrm{X}_{2}^{2}$

$\mathrm{Y}_{2}=2.67-4.93 * \mathrm{X}_{1}+6.25 * \mathrm{X}_{1} \mathrm{X}_{2}-0.167 * \mathrm{X}_{1}^{2}$

Response surface graphs were generated using above polynomial equations, which represents simultaneous effect of one variable on response parameters by taking one variable at a constant level.

The $\mathrm{P}$ value (5\% level) and the value of correlation coefficient $\left(\mathrm{r}^{2}\right)$ obtained for effect on physical appearance was 0.0050 and 0.9119 indicating a good fit of the model. The counter plot for the effect on physical appearance (Figure 4 (A)) shows that the red zone having number 3 is the zone where best results will be obtained then the air flow is 60 and the coating time is $4 \mathrm{~min}$. Moreover, the 3D response surface plot (Figure 4 (B)) suggests that as the coating time increases up to certain extent, good physical appearance was observed. The increase in air flow rate leads to improper physical appearance. Both the combined effect of air flow rate and coating time leads to positive effect on physical appearance.

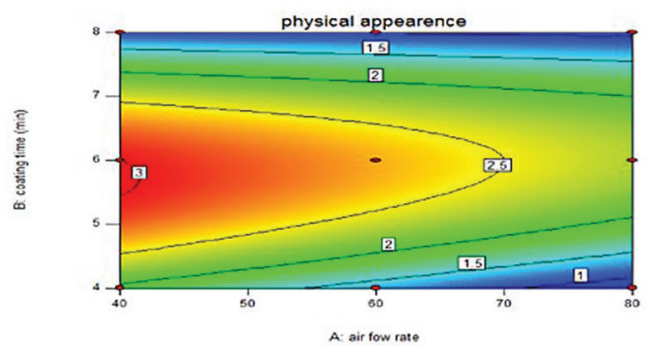

FIGURE 4 - (A) Contour plot (B) Response surface 3D plot; indicating the effect of coating time and airflow rate on physical appearance.

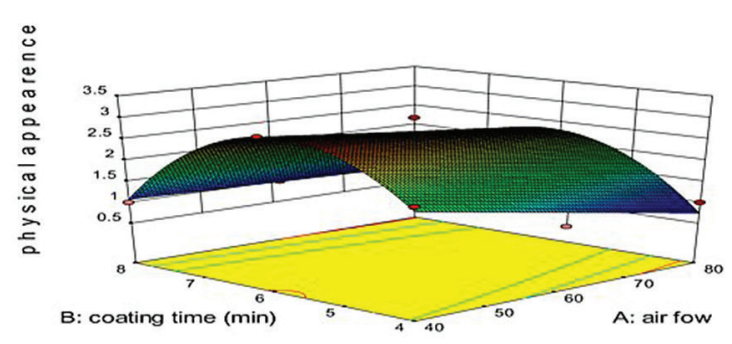

The $\mathrm{P}$ value (5\% level) and the value of correlation coefficient $\left(\mathrm{r}^{2}\right)$ obtained for effect on time taken for $100 \%$ drug release was 0.00381 and 0.9021 indicating a good fit of the model. The counter plot for time taken for $100 \%$ drug release (Figure $5(\mathrm{~A})$ ) shows that the green zone having centre spot is the zone where best results will be obtained then the air flow is 60 and the coating time is 4 min. The 3D response surface plot (Figure 5 (B)) suggests that as the coating time increases up to certain extent, leads to increase in time taken for $100 \%$ drug release. The increase in air flow rate up to certain extent leads to an increase in the time taken for $100 \%$ drug release. It can be concluded that the air flow rate $\left(\mathrm{X}_{1}\right)$ has positive effect on time taken for $100 \%$ drug release. While coating time $\left(\mathrm{X}_{2}\right)$ does not show much effect on it. Both the combined effect of air flow rate and coating time leads to positive effect on physical appearance and the time taken for $100 \%$ drug release. In order to validate the equation that describe the influence of independent parameters on time taken for $100 \%$ drug release and physical appearance, the additional two check point experiments were performed (Table VII) . The t-test was also applied between the actual value and predicted value of dependent parameters. From the results, it was found that there were excellent agreement between the measured response and predicted response by mathematical data. The differences between measured and predicted values were not found to be statistically significant.

Thus, it can be concluded that the polynomial equations fits the data satisfactorily and were valid for predicting the time taken for $100 \%$ drug release and physical appearance for the enteric coated MTX pellets. Thus, considering the results from $3^{2}$ full factorial design, the batch B5 was selected as the optimized batch.

\section{Evaluation of the optimized batch of coated methotrexate pellets}

The optimized batch B5 was evaluated for various parameters like micromeritic parameters, moisture 

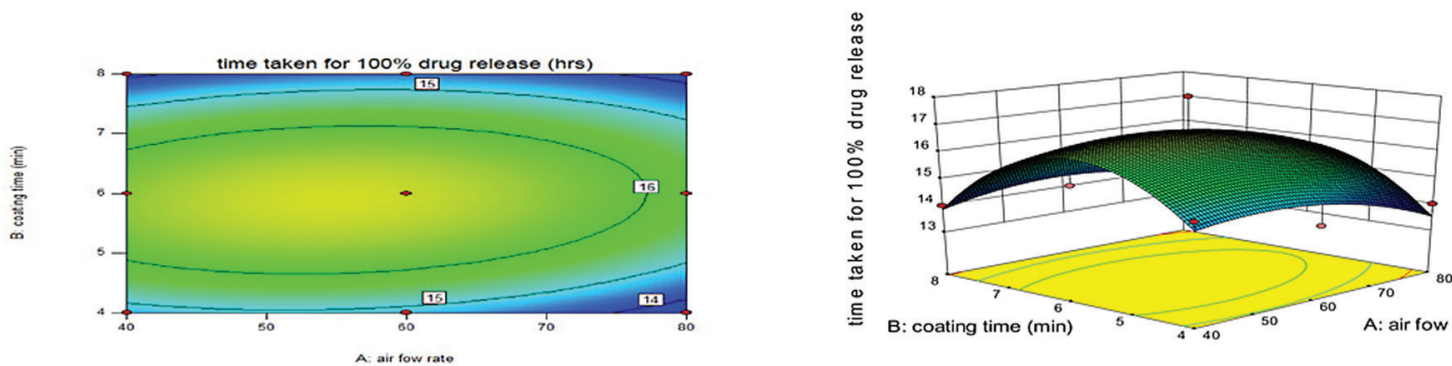

FIGURE 5 - (A) Contour plot (B) Response surface 3D plot; indicating the effect of coating time and airflow rate on time required for $100 \%$ drug release.

TABLE VII - Actual and predicted value of physical appearance and time for $100 \%$ drug release for check point analysis $(\mathrm{n}=3$ )

\begin{tabular}{ccccc}
\hline \multirow{2}{*}{ Batch } & \multicolumn{2}{c}{ Time taken for 100\% drug release (h) } & \multicolumn{2}{c}{ Physical appearance* $^{*}$} \\
\cline { 2 - 5 } & Predicted & Actual & Predicted & Actual \\
\hline 1 & 16.53 & $16 \pm 0.12$ & 1.3 & $1 \pm 0.0$ \\
2 & 16.01 & $16 \pm 0.41$ & 1.09 & $1 \pm 0.0$ \\
\hline
\end{tabular}

*Physical appearance: 1-Aggregated pellets, 2- Average coating with some aggregated pellets, 3- Properly coated pellets with no aggregates

content, sphericity index, drug loading and $\%$ in-vitro drug release study. From the table VIII, it can be concluded that the optimized batch B5 exhibited micromeretic parameters in the acceptable range. The sphericity index was near to 1 which indicated appropriate sphericity of the pellets. It can also be confirmed by the change in the colour of the pellets indicating that they have taken up the coating solution and got coated evenly throughout the surface (Figure 6). The drug loading was also in the desired range and the drug release was sustained up to $17 \mathrm{~h}$ which was almost near to the complete residence time in colon (Figure 7). Thus,

TABLE VIII - Results of evaluation parameters of optimized batch of methotrexate coated pellets (Batch B5)

\begin{tabular}{lc}
\hline Evaluation Parameters & Results \\
\hline Bulk density $(\mathrm{g} / \mathrm{mL})$ & $0.667 \pm 0.09$ \\
Tapped density $(\mathrm{g} / \mathrm{mL})$ & $0.769 \pm 0.08$ \\
Carr's Index $(\%)$ & $13.29 \pm 0.07$ \\
Hausner's ratio & $1.15 \pm 0.06$ \\
Angle of repose $(\theta)$ & $30.73 \pm 0.05^{\circ}$ \\
Moisture content $(\%)$ & $0.058 \pm 0.1$ \\
Sphericity index & $0.943 \pm 0.06$ \\
Friability $(\%)$ & $0.423 \pm 0.09$ \\
Drug loading $(\%)$ & $99.58 \pm 0.03$ \\
Time required for $100 \%$ drug & $17 \pm 0.07$ \\
release (h) & \\
\hline
\end{tabular}

it can be concluded that the optimized batch B5 fulfilled all the desired criteria.
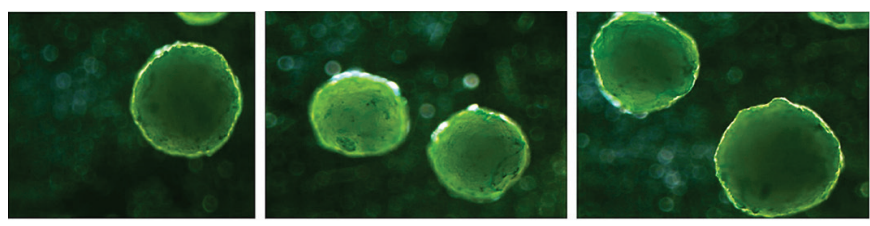

FIGURE 6 - Images of methotrexate coated pellets.

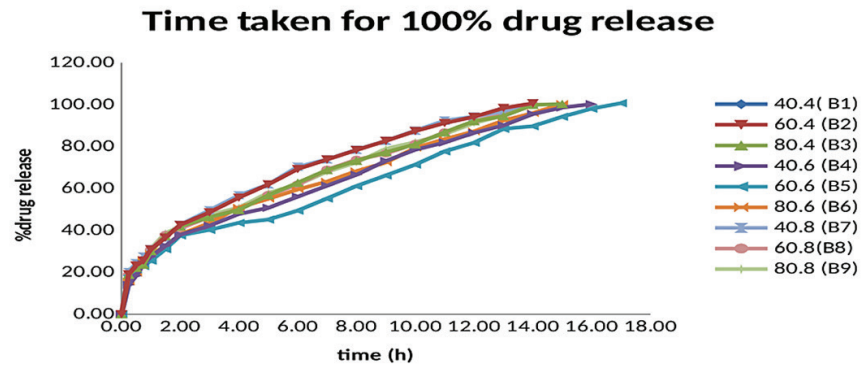

FIGURE 7 - Time taken for 100\% drug release for methotrexate coated pellets.

\section{Ex-vivo study}

The result of the ex-vivo study indicated that 100\% drug release was obtained within $24 \mathrm{~h}$ as that of the residence time for colon. The $71.76 \%$ drug release of the optimized batch B5 observed during ex-vivo study was equivalent to $71.35 \%$ drug release during in vitro study 
at the $10^{\text {th }} \mathrm{h}$. (Figure 8). Moreover, when the drug release data were fitted to various kinetic models, it followed Higuchi model indicating the diffusion mechanism of the drug release from the matrix (Fick's Law).

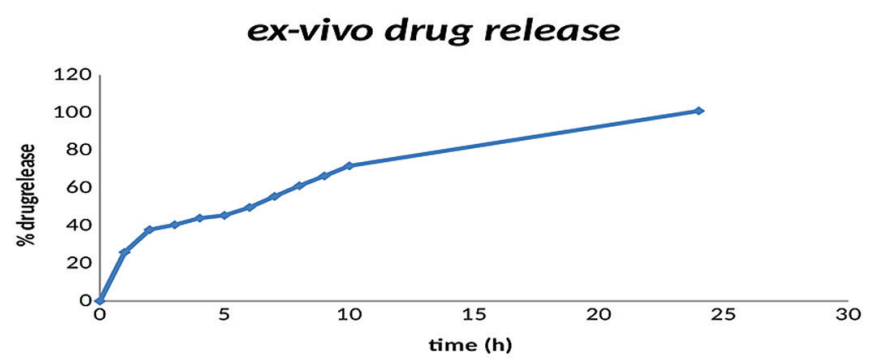

FIGURE 8 - Ex-vivo drug release of optimized batch of methotrexate coateds pellets.

\section{CONCLUSION}

Colon cancer is the second most common cause of mortality in cancer. The chemotherapeutic agentMethotrexate is the drug of choice which inhibits the thymidylate synthesis and thereby arrests the cell growth in S-phase. But, methotrexate gets metabolized in the stomach when given orally and does not reach the specific site to provide proper treatment. Due to the unwanted metabolism, hepatotoxicity and nephrotoxicity are prominently seen. The dosing of this drug is thrice a day and its half life is 3-4 hrs. Sustained release formulation has not been developed yet for methotrexate. Hence, the aim of the study was to develop delayed release pellets of methotrexate for industrial application, cost effectiveness and sustaining the drug release at colonic site. The preformulation study was performed in order to characterize the purity of the drug and to check drugexcipients compatibility. Selection of excipients was performed thorough literature review and screening methods. The results were complied with the standard reported values in the literature and revealed that drug and excipients were compatible with each other.

Based on the literature search and wide industrial applicability, Extrusion-spheronization was selected to prepare pellets. By using Microcrystalline cellulose (MCC) (Avicel PH 101) (spheronization aid), Ethyl Cellulose (release retardant) and Coating polymer (Eudragit S 100) sustained release pellets were developed. The process parameters like spheronization speed, spheronization time, drying time and the formulation related parameters like selection of granulating fluid and its amount, concentration of ethyl cellulose were optimized. The effect of different formulation parameters and process parameters on physical appearance, micromereic parameters, sphericity index, friability, drug loading and the $\%$ in vitro drug release were also studied. Batch P17 with ethyl cellulose: MCC ratio of 3:7 was obtained as the optimized ratio for the core pellets. Based on preliminary study and literature review, coating time and coating air flow rate were selected as independent variables in $3^{2}$ full experimental designs as these two parameters significantly affect physical appearance and $\%$ in vitro drug release of pellets. Polynomial equations by statistical evaluation of the results were obtained in order to correlate the independent and dependent variables. Desirability function was utilized to obtain the optimized batch. Based on the overall desirability factor and design expert software the optimized batch was selected. To achieve site specific drug release, fabricated fluid bed coater was used to coat all the batches of methotrexate pellets using Eudragit S100 as coating polymer. The batch B5 containing ethyl cellulse:MCC 3:7 ratio formulated with coating time of 6 min and coating air flow of $60 \mathrm{~cm}^{3} / \mathrm{mL}$ proved to be optimized batch. The $\%$ in vitro drug release study was performed by change over media in which initially $0.1 \mathrm{~N} \mathrm{HCl}$ was used followed by phosphate buffer $\mathrm{pH} 6.8$ and finally phosphate buffer $\mathrm{pH}$ 7.4 wherein formulation exhibited sustained release effect till $17 \mathrm{~h}$. The ex-vivo study was performed in rat colon and different kinetic models were applied to check the release mechanism of pellets which indicated that coated pellets followed Higuchi diffusion mechanism.

Thus, all the results obtained while development of the dosage form were as per the desired set of the objectives. Therefore, the coated pellets can be a good candidate for site specific delivery of MTX to colon by decreasing the gastric irritation, reducing dose frequency and by improving patient compliance for the treatment of colon cancer. Though, the in-vivo study should be performed using suitable animal model to demonstrate the enhancement in bioavailability and site specific action of formulation.

\section{CONFLICT OF INTEREST}

The authors report no conflict of interest.

\section{ACKNOWLEDGEMENT}

We are thankful to Sun Pharmaceuticals Pvt. Ltd. (SPARC, Baroda, Gujarat) for providing the gratis sample of Methotrexate and also thankful to Primex (Norway, Iceland) and Evonik Degussa (India) Pvt. Ltd. (Mumbai, Maharashtra) for providing gratis samples of Chitoclear FG95 and Eudragit polymers respectively. We extend our thanks to Dr. Ravish Patel and Dr. Amit Patel, RPCP, 
CHARUSAT for helping us to work on modified fluid bed coater.

\section{REFERENCES}

Akhgari A, Sadeghi F, Garekani HA. Combination of timedependent and $\mathrm{pH}$-dependent polymethacrylates as a single coating formulation for colonic delivery of indomethacin pellets. Int J Pharm. 2006; 320(1):137-42.

Gandhi R, Kaul CL, Panchagnula R. Extrusion and spheronization in the development of oral controlled-release dosage forms. Pharm Sci Techno Today. 1999; 2(4):160-70.

Gupta N, Gowda DV, Balamuralidhara V, Khan M. Formulation and evaluation of olanzapine matrix pellets for controlled release. Daru. 2011; 19(4):249-56.

He W, Du Q, Cao DY, Xiang B, Fan LF. Study on colon-specific pectin/ethylcellulose film-coated 5-fluorouracil pellets in rats. Int J Pharm. 2008;348(1):35-45.

Hileman GA, Upadrashta SM, Neau SH. Drug solubility effects on predicting optimum conditions for extrusion and spheronization of pellets. Pharm Develop Technol. 1997;2(1):43-52.

Jia J, Dong C, Zhang W, Cui Y, Liu J. Evaluation of pharmacokinetic and pharmacodynamic relationship for oral sustained-release atenolol pellets in rats. J Pharm Biomed Anal. 2011;55(2):342-8.
Kaynak MS, Kas HS, Oner L. Formulation of controlled release glipizide pellets using pan coating method. Hacettepe Univ J Faculty Pharmacy. 2007;27(2):93-106.

Omwancha WS, Mallipeddi R, Valle BL, Neau SH. Chitosan as a pore former in coated beads for colon specific drug delivery of 5-ASA. Int J Pharm. 2013;441(1):343-51.

Priese F, Frisch T, Wolf B. Comparison of film-coated retarded release pellets manufactured by layering technique or by bed rotor pelletization. Pharm Develop Technol. 2015;20(4):417-25.

Sogali BS, Yousuff M, Nayak S. Influence of natural gums for effective colon targeting of methotrexate for the treatment of colorectal cancer. Int J Pharmacy. 2012;2:498-506.

Sriamornsak P, Nunthanid J, Luangtana-Anan M, Weerapol Y, Puttipipatkhachorn S. Alginate-based pellets prepared by extrusion/spheronization: effect of the amount and type of sodium alginate and calcium salts. Eur J Pharm Biopharm. 2008;69(1):274-84.

Steckel H, Mindermann-Nogly F. Production of chitosan pellets by extrusion/spheronization. Eur J Pharm Biopharm. 2004;57(1):107-14.

Vervaet C, Baert L, Remon JP. Extrusion-spheronisation: A literature review. Int J Pharm. 1995; 116(2):131-46.

Received for publication on $01^{\text {st }}$ June 2017 Accepted for publication on $20^{\text {th }}$ March 2018 